\title{
Atomic spectroscopy S \\ Measurement of Flame Temperature Using Simultaneous Dual- line Atomic Absorption of Two Elements
}

\author{
Yanqi Xiong, ${ }^{\mathrm{a}, \dagger}$ Tao Lin, ${ }^{\mathrm{a}, \dagger}$ Xiaoming Jiang, ${ }^{\mathrm{b}}$ and Xiandeng Hou ${ }^{\mathrm{a}, \mathrm{b}, *}$ \\ a College of Chemistry, Sichuan University, Chengdu, Sichuan 610064, P.R. China \\ b Analytical \& Testing Center, Sichuan University, Chengdu, Sichuan 610064, P.R. China
}

Received: 15 August 2020, Revised: 7 September 2020, Accepted: 7 September 2020, Available online: 10 September 2020.

DOI: $10.46770 / A S .2020 .05 .003$

ABSTRACT: By use of dual atomic absorption lines with a two-element hollow cathode lamp, a simple method was developed for air/acetylene flame temperature measurement. The results are comparable to literature values with a peak temperature at about $2400 \mathrm{~K}$. The spatial temperature variation of a fuel-rich flame was investigated, and it was found that the tendency for variation conformed to the flame combustion process. The proposed method was compared to the traditional dual-line atomic absorption method of indium and showed a similar tendency of temperature variation. The proposed instrumental setup is simple, which uses a $\mathrm{Cu} / \mathrm{Fe}$ two-element hollow cathode lamp, a handheld charge coupled device detector, an optical fiber and a computer. Due to its simplicity, non-contact and portability, this technique is promising for flame temperature characterization of various kinds of flames or electrothermal atomizers.

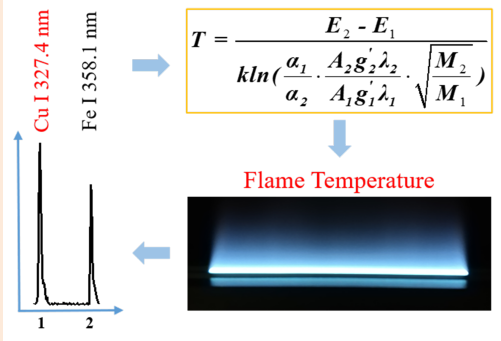

\section{INTRODUCTION}

Flame atomic absorption spectroscopy (FAAS) has been widely employed in various fields for elemental content quantification due to its good sensitivity, high selectivity and low cost of operation. Although FAAS has several shortcomings in comparison to ICP-based techniques, such as narrow linear dynamic range, it can satisfy general analytical requirements with the limit of detection at sub-ppm levels for most detectable elements. ${ }^{1,2}$ Measurement of the temperature for exploring the chemical behavior of an analyte in different types of flames, such as atomization processes and atomization efficiencies, is crucial for improving the analytical performance, especially when the flame atomizer is modified in combination with other techniques, ${ }^{3,4}$ and because its properties are to some extent related to the temperature directly or indirectly. Therefore, flame temperature is a vital parameter for FAAS and is defined as the exterior characterization of an average kinetic energy of the flame. ${ }^{5,6}$

Measurement of flame temperature is always an important research topic for physicists and spectrochemists. Although several methods have been developed for this purpose, they can mainly be divided into physical and optical methods. Therein, the thermocouple dominates the physical methods, but a thermocouple needs to be inserted into the flames which will disturb the flame combustion process. As for the optical methods, several atomic spectroscopy methods have been developed. These are mainly based on the thermometric atoms distribution and obey the Boltzmann distribution under local thermodynamic equilibrium (LTE) of a certain element at different energy levels of the atomization, such as a flame. ${ }^{6}$ A premixed laminar air/acetylene flame is considered to follow the LTE conditions based on frequent collision of the particles in the flames, such as electrons, ions, atoms, molecules, and so on. ${ }^{7,8}$ Therefore, the flame temperature can be deduced by dual-line atomic spectroscopy methods involving two different transitions with different excitation energies.

Atomic spectroscopy-based methods for temperature measurement can be divided into a dual-line atomic absorption method, ${ }^{9}$ a dual-line atomic emission method, ${ }^{10}$ and a dual-line atomic fluorescence method. ${ }^{11}$ These methods have been successfully applied for flame characterization, albeit with some deficiencies. For example, an atomic absorption spectroscopy method is normally coupled with a hollow cathode lamp. However, 
this will lead to the less populated lines having higher sensitivity errors because of the difference in exterior radiation intensity at the corresponding wavelength and the varied atomic concentration in the local flame volume. ${ }^{12}$ For atomic emission spectroscopy or atomic absorption methods, the selected lines may also suffer from spectral interferences of background emission of the flame and results in inaccurate measurements. ${ }^{13}$ The dual-line atomic fluorescence spectroscopy method used as a popular flame diagnostic technique is susceptible to the scattering of soot in the flames. ${ }^{14,15}$

For traditional dual-line atomic absorption spectroscopy methods, the flame temperature is characterized by the atomic absorption of two atomic lines under different lower energy levels of one kind of certain thermometric atom. ${ }^{9,}{ }^{12}$ In this report, we propose a new flame temperature measurement method based on the atomic absorption of two thermometric species. The experimental setup is simple and composed of a two-element hollow cathode lamp, a handheld charge-coupled device detector, an optical fiber and a laptop computer to achieve the simultaneous measurement of two atomic absorption lines from two elements. The flame temperature of the air/acetylene flame was then determined and compared with the traditional indium dual-line atomic absorption method. A spatial-resolved temperature profile of the air/acetylene flame and flames with different air/acetylene ratios was also obtained.

\section{EXPERIMENTAL}

Materials and reagents. All reagents used in this work were at least of analytical grade. Acetylene gas (99.99\%) was purchased from Qiaoyuan Gas Co., Ltd. (Chengdu, P.R. China). Deionized water $(18.2 \mathrm{M} \Omega . \mathrm{cm})$ was produced from a water purification system (Pincheng Technology Co., Ltd., Chengdu, P.R. China). Nitric acid was purchased from Suzhou Crystal Clear Co., Ltd. Stock solutions of $\mathrm{Fe}, \mathrm{Cu}$, and $\mathrm{In}$ were prepared from $\mathrm{Fe}\left(\mathrm{NO}_{3}\right)_{3} \cdot 9 \mathrm{H}_{2} \mathrm{O}$ (Shanghai Aladdin Bio-Chem Technology Co., Ltd., P.R. China), $\mathrm{Cu}\left(\mathrm{NO}_{3}\right)_{2} \cdot 3 \mathrm{H}_{2} \mathrm{O}$ (Kelong Chemical Reagent Plant Co., Ltd., P.R. China), and $\mathrm{In}\left(\mathrm{NO}_{3}\right)_{3} \cdot \mathrm{xH}_{2} \mathrm{O}$ (Shanghai Aladdin Bio-Chem Technology Co., Ltd., P.R. China). The test solutions were prepared in $2 \%(\mathrm{v} / \mathrm{v})$ nitric acid, and the concentrations of the test solutions of the thermometric species were in linear dynamic range.

Apparatus. The flame atomizer unit (slot burner, $100 \mathrm{~mm}$ in length) from a commercial flame atomic absorption spectrometer (GGX-600 AAS, HaiGuang instrument Co., Ltd., China) was equipped with a handheld CCD detector (Maya 2000 PRO, 175$401 \mathrm{~nm}$, Ocean Optics, U.S.) or a CCD detector (Maya 2000 PRO, 400-600 nm, Ocean Optics, U.S.) when the indium atomic absorption spectroscopy method was used for flame temperature measurements. The primary radiation source was a $\mathrm{Cu} / \mathrm{Fe}$ twoelement hollow cathode lamp, and the multi-wavelength radiation

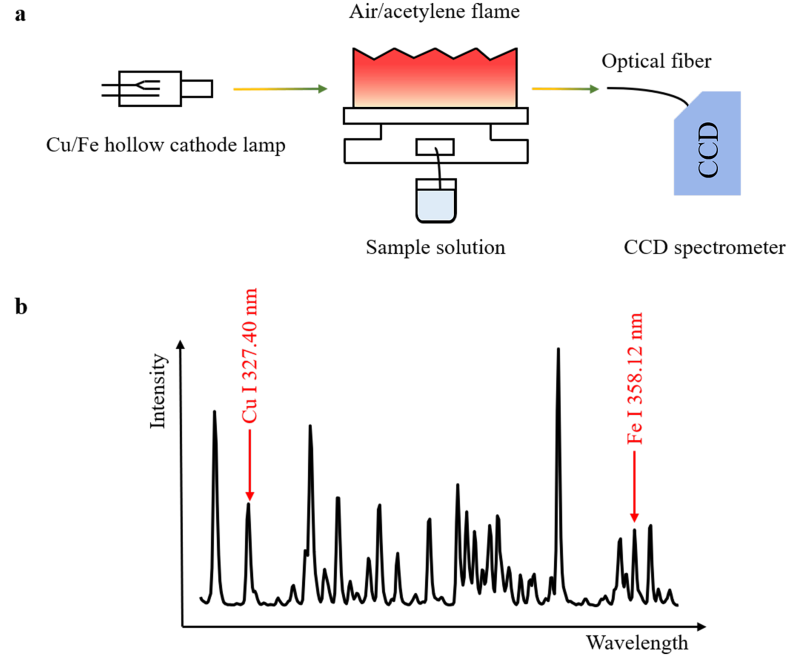

Fig. 1 Schematic diagram of experimental setup for flame temperature measurement (a); and simultaneous detection of the intensities of the spectral lines of $\mathrm{Cu}$ I $327.40 \mathrm{~nm}$ and $\mathrm{Fe}$ I $358.12 \mathrm{~nm}$ from a $\mathrm{Cu} / \mathrm{Fe}$ hollow cathode lamp for atomic absorbance calculation (b).

intensity was simultaneously detected by the CCD detector coupled with an optical fiber (QP600-025-XSR, 180-800 nm, Ocean Optics, U.S.) as illustrated in Fig. 1. The premixed laminar chemical flame was composed of an air and acetylene gas mixture. A commercial premixed spray chamber also from the GGX-600 AAS was utilized to premix the sample aerosol, air and acetylene. In order to keep the injection velocity of the sample aerosol constant, the gas mixture was varied by only changing the acetylene gas flow rate.

\section{RESULTS AND DISCUSSION}

\section{Flame temperature equations with a pair of $\mathrm{Cu} / \mathrm{Fe}$ atomic absorption lines}

An accurate profile of flame temperature is a challenge and the dual-line atomic absorption method was first proposed by L'vov et al. in $1971 .{ }^{12}$ The Boltzmann distribution was utilized to build equations relating to atomic absorption intensities of a pair of spectral lines with different lower levels of excitation energies. Indium was used for temperature determination based on the atomic absorption at 410.18 and $451.13 \mathrm{~nm}$ as proposed by Browner and Winefordner in 1972, ${ }^{16}$ and the latest more accurate parameters were updated in a calculation as described in Eq. 1.

$$
T=-\frac{1383}{\lg \left(0.438 \times \frac{\alpha_{2}}{\alpha_{1}}\right)}
$$

where $\alpha$ is the atomic absorbance, $T$ is the calculated flame temperature, the subscripts 1 and 2 represent the spectral lines of indium at 410.18 and $451.13 \mathrm{~nm}$, respectively.

For atomic spectroscopy-based methods, a certain thermometric species is utilized, but it would lead to a higher sensitivity error 
in lower populated levels. ${ }^{12}$ For a dual-line atomic emission method, a different equation based on the optical emission intensities of the line pair from two different elements, $\mathrm{Rb} / \mathrm{Li}$ or $\mathrm{Li} / \mathrm{Ba}$, was proposed by Donati and Jones in $2011 .{ }^{10}$ By controlling the concentration of two elements in a test solution, the same number of atoms could be realized in a local flame by using the Boltzmann distribution under thermodynamic equilibrium. ${ }^{10}$

In this study, a new flame temperature equation for two elements was established based on the absorbance ratio of a pair of spectral lines. Based on the atomic absorbance ratios of the spectral lines of two elements (Eq. 2 and Eq. 4) and the partition function, the flame temperature could be calculated based on the atomic absorbance of the different elements (Eq. 5). In order to simplify the calculation, only Doppler broadening was considered, and since the selected line pair was close, the error could be ignored from the Lorentz broadening.

$$
\begin{aligned}
& \alpha_{1}=\lg \frac{I_{B l a n k}}{I_{\text {Sample }}}=0.4343 \frac{2 \sqrt{\pi \ln 2}}{\Delta \lambda_{D_{1}}} \cdot \frac{e^{2}}{m_{e} c} \cdot \frac{g_{1}}{Q_{t}} \cdot \exp \left(-\frac{E_{1}}{k T}\right) \boldsymbol{N} \boldsymbol{f}_{1} L \\
& \Delta \lambda_{D}=7.16 \times 10^{-7} \lambda \sqrt{\frac{T}{M}} \\
& \frac{\alpha_{1}}{\alpha_{2}}=\frac{\lambda_{2} \sqrt{\frac{T}{M_{2}}}}{\lambda_{1} \sqrt{\frac{T}{M_{1}}}} \cdot \frac{\exp \left(-\frac{E_{1}}{k T}\right) f_{1} g_{1}}{\exp \left(-\frac{E_{2}}{k T}\right) f_{2} g_{2}} \\
& T=\frac{E_{2}-E_{1}}{k \ln \left(\frac{\alpha_{1}}{\alpha_{2}} \cdot \frac{A_{2} g_{2}^{\prime} \lambda_{2}}{A_{1} g_{1}^{\prime} \lambda_{1}} \cdot \sqrt{\frac{M_{2}}{M_{1}}}\right)}
\end{aligned}
$$

where $\alpha$ is the atomic absorbance, $I_{\text {Blank }}$ and $I_{\text {Sample }}$ are the radiation intensities of the atomic lines from the lamp with blank and sample solution introduced into the flame, $k$ is the Boltzmann constant, $E$ is the excitation energy, $L$ is the absorption path length, $\lambda$ is the absorption wavelength, $M$ is the standard atomic mass, $\Delta \lambda_{D}$ is the Doppler broadening, $e$ is the charge of the electron, $m_{e}$ is the mass of the electron, $c$ is the velocity of light, $g$ and $g^{\prime}$ are the statistical weights corresponding to the lower and upper level of the spectral line, $Q_{t}$ is the partition function, $f$ is the oscillator strength, $N$ is the concentration of the free atoms of an element, $A$ is the Einstein transition probability, $T$ is the calculated flame temperature. The subscripts 1 and 2 represent elements 1 and 2, respectively.

In comparison with the traditional dual-line atomic absorption method, by selecting spectral lines with similar primary radiation intensities of two elements, such as $\mathrm{Cu}$ I $327.40 \mathrm{~nm}$ and Fe I $358.12 \mathrm{~nm}$ in this study, the difference in sensitivity error for the target spectral lines could be reduced. The parameters of these spectral lines used in the equations are summarized and listed in Table $1 .{ }^{17,18}$

\section{Flame temperature of different air/acetylene ratios}

The proposed method was utilized to measure the flame
Table 1. Parameters of Spectral Lines for the Flame Temperature Measurement and Calculation

\begin{tabular}{lcccc}
\hline $\begin{array}{l}\text { Wavelength } \\
(\mathbf{n m})\end{array}$ & $\begin{array}{c}\text { Lower level } \\
\left(\mathbf{c m}^{-1}\right)\end{array}$ & $\begin{array}{c}\text { Upper level } \\
\left(\mathbf{c m}^{-1}\right)\end{array}$ & $\begin{array}{c}\text { gA } \\
\left(\mathbf{s}^{-1}\right)\end{array}$ & M \\
\hline Cu I 327.40 & 0.000 & 30535.324 & $2.752 \times 10^{8}$ & 63.546 \\
Fe I 358.12 & 6928.268 & 34843.957 & $1.326 \times 10^{9}$ & 55.845 \\
\hline
\end{tabular}

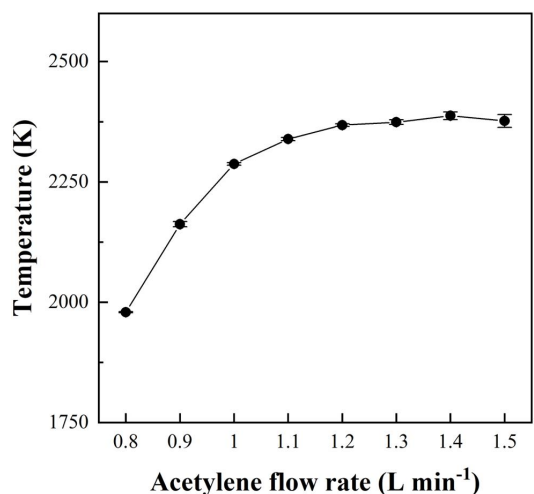

Fig. 2 Flame temperature versus gas flow rate of acetylene in an air/acetylene chemical flame. The error bars represent standard deviations of triplicate temperature measurements, and the same below.

temperature of a premixed air/acetylene chemical flame. In order to use Eq. 5 for the temperature measurement, the two elements need to have the same number of atoms in the local flame. Considering the difference in the atomization efficiencies of these two elements and their concentration in the test solution, the detected absorbance ratios should be combined with the relative atomization efficiency and the number of moles, and the relative atomization efficiency of $\mathrm{Fe}$ and $\mathrm{Cu}$ calculated using the method proposed by Zeegers et al. ${ }^{19}$ During the flame temperature measurement, therefore, a test solution containing $\mathrm{Cu}$ of $5.16 \mu \mathrm{g}$ $\mathrm{mL}^{-1}$ and $\mathrm{Fe}$ of $40.0 \mu \mathrm{g} \mathrm{mL} \mathrm{m}^{-1}$ was used.

The flame temperature is mainly dependent on the gas flow rate of the oxidant and fuel and the stoichiometric ratio of the oxidant and fuel gas. In this case, the relation between the flame temperature and the gas flow rate of acetylene is shown in Fig. 2, where the acetylene flow rates were changed from 0.8 to $1.5 \mathrm{~L} \mathrm{~min}^{-1}$ with the air flow rate kept constant at $6.0 \mathrm{~L} \mathrm{~min}^{-1}$ under the same observation height above the burner $(6 \mathrm{~mm})$. All these flames were in fuel-excess conditions where for a stoichiometric air/acetylene flame of complete combustion the gas flow rate is about $0.48 \mathrm{~L}$ $\mathrm{min}^{-1}$ for acetylene to air gas flow rate of $6.0 \mathrm{~L} \mathrm{~min}^{-1}$ (with ca. $20 \%$ (v/v) oxygen in air). With an increase in acetylene gas flow rate, the flame temperature increased slightly from ca. $1980 \mathrm{~K}$ with the acetylene flow rate at $0.8 \mathrm{~L} \mathrm{~min}^{-1}$ to ca. $2390 \mathrm{~K}$ with acetylene flow rate at $1.5 \mathrm{~L} \mathrm{~min}^{-1}$, as shown in Fig. 2. A possible explanation for this phenomenon could be that the unreacted acetylene will combust with the surrounding air, thus resulting in an increase in flame temperature. These flame temperatures were comparable to the values in the literature as the normally recorded temperature is about 2400 to $2600 \mathrm{~K}$. , 10, 20 The flame temperature is mainly dependent on the type of flame, the gas flow rates, the burner 
design, the sampling method and the optics of measuring method. Therefore, the flame temperature measured and calculated by the proposed method is acceptable.

\section{Flame temperature at different burner height}

The dependence of flame temperature on the height above the burner is illustrated in Fig. 3. Considering the spot size of the primary light source, the spatial resolution was set as $2 \mathrm{~mm}$ for this study. The chemical flame was composed of $1.0 \mathrm{~L} \mathrm{~min}^{-1}$ acetylene and $6.0 \mathrm{~L} \mathrm{~min}^{-1}$ air. The flame temperature showed a broad peak at $6 \mathrm{~mm}$ with a flame temperature of $2290 \mathrm{~K}$, then decreased with the burner height at $20 \mathrm{~mm}$ to ca. $1780 \mathrm{~K}$. The main reason for this result is that with an increase in burner height, less acetylene gas will participate in the chemical combustion process because the chemical reaction rate is faster than the gas flow rate. ${ }^{21}$

\section{Flame temperatures measured by different methods}

Flame temperatures obtained by the proposed method were compared with the traditional indium dual-line atomic absorption method ${ }^{16}$ under the same chemical flame conditions, the results are presented in Fig. 4. The air flow rate was set at $6.00 \mathrm{~L} \mathrm{~min}^{-1}$, the observation height at $6 \mathrm{~mm}$, and the acetylene flow rates were varied from 0.8 to $1.5 \mathrm{~L} \mathrm{~min}^{-1}$. The concentration of the indium test solution was $40 \mu \mathrm{g} \mathrm{mL}^{-1}$ and the $\mathrm{Cu} / \mathrm{Fe}$ test solution was the same as above. As shown in Fig. 4, the flame temperatures calculated from the equation of the indium traditional dual-line atomic absorption method were from ca. $2750 \mathrm{~K}$ with acetylene of $0.8 \mathrm{~L}$ $\mathrm{min}^{-1}$ to ca. $2930 \mathrm{~K}$ with acetylene of $1.5 \mathrm{~L} \mathrm{~min}^{-1}$, with about 540 to $770 \mathrm{~K}$ higher than the $\mathrm{Cu} / \mathrm{Fe}$ double-element atomic absorption method and 300-500 K higher than the literature values. ${ }^{10,20}$ The reason for these differences may be due to an imprecise determination of the atomization efficiencies and the differences in the photoelectric response coefficients of the spectrometer at different wavelengths. Fortunately, the variation tendency between the flame temperature and the acetylene gas flow rates of these two methods was found to be similar, probably suggesting that the proposed method would be an interesting alternative for the traditional dual-line atomic absorption method.

\section{CONCLUSIONS}

A flame temperature measurement technique was developed based on dual-line atomic absorption spectroscopy of $\mathrm{Cu} / \mathrm{Fe}$ using a two-element hollow cathode lamp, together with a CCD spectrometer for simultaneous detection. The proposed method was successfully applied for the temperature characterization of an air/acetylene chemical flame. The flame temperatures of different gas flow rates and observation heights above the burner were measured/calculated by this method and found to be comparable with the literature values. In comparison with the traditional dual-line atomic absorption method, a much smaller error would be introduced by subjectively selecting two spectral lines under different lower energy levels with similar radiation

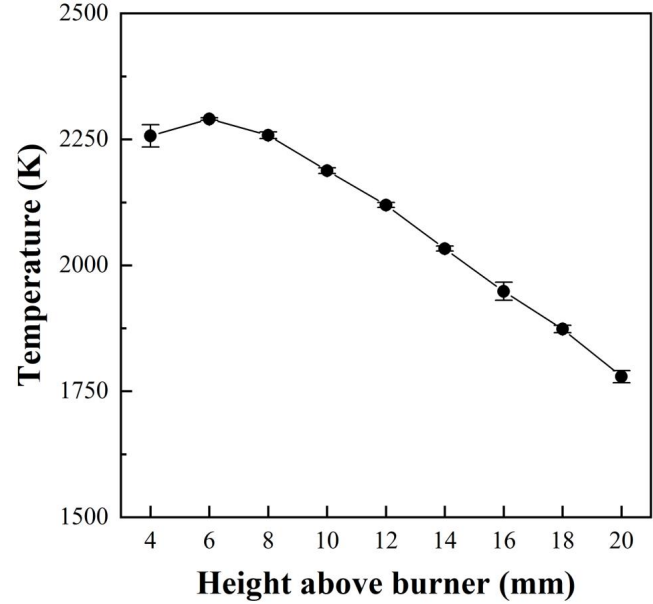

Fig. 3 Flame temperature with observation height in air/acetylene chemical flame.

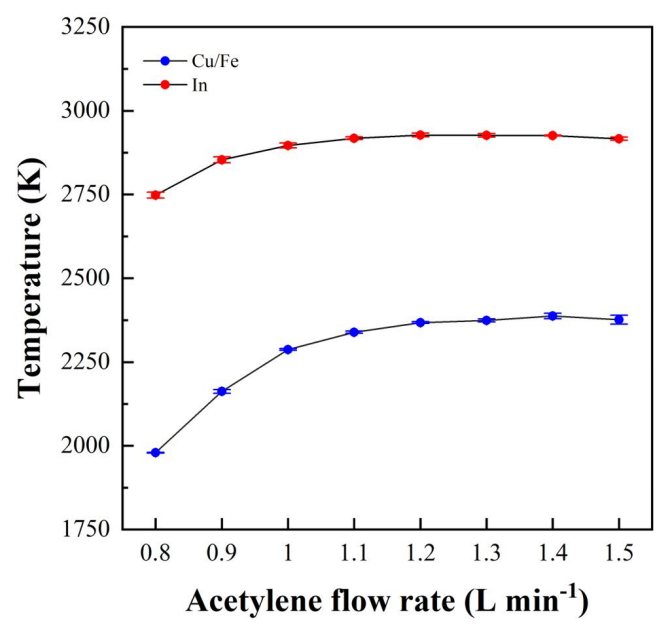

Fig. 4 Comparison of flame temperatures obtained by traditional indium dual-line atomic absorption method (red dots) and $\mathrm{Cu} / \mathrm{Fe}$ two-element atomic absorption method (blue dots).

intensities, and selecting the wavelengths of the two elements to be as close as possible to each other, although an accurate atomization efficiency rate of the thermometric species is indispensable. The proposed method is an interesting and promising alternative to the conventional dual-line atomic absorption method for the temperature measurement of various flames.

\section{AUTHOR INFORMATION}

\section{Corresponding Author}

*X.-D. Hou

Email address: houxd@scu.edu.cn

\section{Notes}

†Y.-Q. Xiong and T. Lin contributed equally to this work.

The authors declare no competing financial interest. 


\section{ACKNOWLEDGMENTS}

The authors gratefully acknowledge the financial support for this work from the Ministry of Education through the 111 Project (Grant No. B17030) and the Fundamental Research Funds for the Central Universities.

\section{REFERENCES}

1. E. H. Evans, J. Pisonero, C. M. M. Smith and R. N. Taylor, J. Anal. At. Spectrom., 2020, 35, 830-851. http://doi.org/10.1039/D0JA90015J

2. M. Khan and M. Soylak, At. Spectrosc., 2018, 39, 81-89. http://doi.org/10.46770/AS.2018.02.005

3. E. G. Bakirdere and E. Maltepe, At. Spectrosc., 2019, 40, 139-144. http://doi.org/10.46770/AS.2019.04.005

4. A. A. Brown and A. Taylor, Analyst, 1984, 109, 1455-1459. http://doi.org/10.1039/AN9840901455

5. Y. Toya, T. Itagaki and K. Wagatsuma, Spectrochim. Acta Part B: At. Spectrosc., 2016, 125, 146-151. https://doi.org/10.1016/j.sab.2016.10.005

6. G. Sutton, A.M. Fateev, and A. Rodríguez-Conejo, Int. J. Thermophys., 2019, 40, 99. https://doi.org/10.1007/s10765-019-2557-6

7. J. D. Wang, S. Y. Li, X. M. Wang, W. G. Li, Z. R. Chen, and Y. H. Luo, Spectr. Lett., 1992, 25, 769-775. https://doi.org/10.1080/00387019208020709

8. N. M. Laurendeau, Prog. Energy Combust. Sci., 1988, 40, 147-170. https://doi.org/10.1016/0360-1285(88)90002-0

9. R. E. Sturgeon and C. L. Chakrabarti, Spectrochim. Acta Part B: At. Spectrosc., 1977, 328, 231-255. https://doi.org/10.1016/05848547(77)80008-6
10. G. L. Donati and B. T. Jones, J. Anal. At. Spectrom., 2011, 26, 838-844. https://doi.org/10.1039/C0JA00172D

11. N. Omenetto, P. Benetti, and G. Rossi, Spectrochim. Acta Part B. At. Spectrosc., 1972, 27, 453-461.

https://doi.org/10.1016/0584-8547(72)80044-2

12. B. V. L'vov, D. A. Katskov and L. P. Kruglikova, J. Appl. Spectrosc., 1971, 14, 569-575. https://doi.org/10.1007/BF00605790

13. R. Papai, M. A. S. de Freitas, K. T. da Fonseca, G. A. de Almeida, J. R. F. da Silveira, A. L. N. da Silva, J. B. F. Neto, C. A. L. dos Santos, F. J. G. Landgraf, and M. S. Luz, Anal. Chim. Acta, 2019, 1085, 21-28. https://doi.org/10.1016/j.aca.2019.07.049

14. Z. Sun, Z. Alwahabi, B. Dally, and G. Nathan, Proc. Combust. Inst., 2019, 37, 1417-1425. https://doi.org/10.1016/j.proci.2018.06.127

15. D. H. Gu, Z. W. Sun, G. J. Nathan, P. R. Medwell, Z. T. Alwahabi, B. B. Dally, Combust. Flame, 2016, 167, 481-493. https://doi.org/10.1016/j.combustflame.2015.09.028

16. R. F. Browner and J.D. Wineford. Anal. Chem., 1972, 44, 247-252. http://doi.org/10.1021/ac60310a020

17. A. Kramida, Y. Ralchenko, J. Reader, and NIST ASD Team NIST Atomic Spectra Database (version 5.7.1). https://physics.nist.gov/asd.

18. J. R. D. Laeter, J. K. Böhlke, P. D. Bièvre, H. Hidaka, H. S. Peiser, K. J. R. Rosman, and P. D. P. Taylor, Pure Appl. Chem., 2003, 75, 683-800. https://doi.org/10.1351/pac200375060683

19. P. J. T. Zeegers, Spectrochim. Acta Part B: At. Spectrosc., 1969, 24, 243-254. https://doi.org/10.1016/0584-8547(69)80029-7

20. C. B. Ke and K. C. Lin, Appl. Spectrosc., 1998, 52, 187-194. https://doi.org/10.1021/ac980970t

21. V. I. Babushok and A. W. Miziolek, Combust. Flame, 2004, 136, 141-145. https://doi.org/10.1016/j.combustflame.2003.09.006 